\title{
Coronary Artery Bypass Surgery for Patients with Severe Cerebrovascular Atherosclerosis: Importance of Preoperative Assessment and Perioperative Management ${ }^{*}$
}

\author{
Hajime Imura $^{1 \#, \text { Dai Nishina }}{ }^{2}$, Yuuji Maruyama ${ }^{1}$, Makoto Shirakawa ${ }^{1}$, \\ Motoko Tanoue ${ }^{1}$, Daigo Suzuki ${ }^{1}$, Takahide Yoshio ${ }^{1}$, Masami Ochi ${ }^{2}$ \\ ${ }^{1}$ Department of Cardiovascular Surgery, Nippon Medical School \\ Musashikosugi Hospital, Kawasaki, Japan \\ ${ }^{2}$ Nippon Medical School Hospital, Kawasaki, Japan \\ Email: " himura@nms.ac.jp
}

Received November 7, 2013; revised November 28, 2013; accepted December 5, 2013

Copyright (C) 2013 Hajime Imura et al. This is an open access article distributed under the Creative Commons Attribution License, which permits unrestricted use, distribution, and reproduction in any medium, provided the original work is properly cited. In accordance of the Creative Commons Attribution License all Copyrights (C) 2013 are reserved for SCIRP and the owner of the intellectual property Hajime Imura et al. All Copyright (C) 2013 are guarded by law and by SCIRP as a guardian.

\begin{abstract}
Objective: Cerebrovascular atherosclerosis is known to play a crucial role in perioperative stroke in coronary arterial bypass grafting (CABG). This study is to identify the degree of severity of cerebrovascular lesions for which patients can still undergo CABG with an acceptably low risk in current techniques. Methods: Cerebrovascular atherosclerosis was evaluated and graded for 200 consecutive patients prior to CABG. Grading was initially based on the level of stenosis in carotid, vertebral, and cerebral arteries: grade- 0 : normal or mild stenosis in cerebral arteries or stenosis $<50 \%$ in other arteries; grade-1: moderate in cerebral arteries or 50\% - 69\% in others; grade- 2 : severe in cerebral arteries or $70 \%$ $-89 \%$ in others; grade-3: occlusion in cerebral arteries or $90 \%-100 \%$ in others. The grading was finally adjusted to a risk of regional ischemia by considering symptoms, number of lesions, and brain perfusion in scintigram. Therefore, some patients were up-graded. Off-pump CABG was scheduled for all patients. The lowest systolic arterial pressure during surgery was differently controlled in each grade: grade- $0: \geq 70 \mathrm{mmHg}$; grade- $1: \geq 80 \mathrm{mmHg}$; grade- $2: \geq 80 \mathrm{mmHg}$ with intra-aortic balloon pump (IABP); grade- 3 : $\geq 90 \mathrm{mmHg}$ with IABP; grade-4: $\geq 90 \mathrm{mmHg}$ with IABP and administration of thyamiral. Results: Grade- 1 and -2 included 38 and 29 patients respectively. Grade- 3 initially included 36 patients and 14 of them were up-graded to grade-4 (extremely high risk patients). Stroke was seen in one patient $(0.5 \%)$, for whom mild speaking disturbance occurred on the fifth day from CABG. Conclusion: Patients with severe cerebrovascular atherosclerosis can undergo $\mathrm{CABG}$ with a low risk of stroke. Intraoperative management of blood pressure may be critical for stroke prevention in CABG.
\end{abstract}

Keywords: Coronary Arterial Bypass Grafting; Stroke; Cerebrovascular Disease

\section{Introduction}

Stroke is a critical complication in coronary artery bypass grafting (CABG). Associated with nine-fold increase in mortality, stroke can cause serious physical disability if the patient survives [1]. Numerous studies have investigated this crucial issue and illuminated several risk factors [2]; however, most of those studies (especially large-scale and retrospective studies) neglected

"Conflict of interest: none declared.

${ }^{\#}$ Corresponding author. to include sufficient preoperative cerebrovascular assessment.

While previous studies emphasized the impact of extracranial carotid arterial disease on post-CABG stroke [3], recent studies indicated limited advantage of carotid endarterectomy or stenting prior to $\mathrm{CABG}$ for prevention of stroke [4]. Thus the benefit of carotid revascularization is currently dubious [5,6] and the most current guidelines recommend isolated CABG for this pathology if the carotid disease is unilateral and asymptomatic [7]. In these circumstances, $\mathrm{CABG}$ for patients with severe 
cerebrovascular lesions is expected to increase in future.

Recently, a large scale study reported that atherosclerotic score was the most influencing factor for post-CABG stroke [8]. Importance of sufficient preoperative assessment of intracranial arteries and vertebral-basilar arteries as well as extracranial carotid artery was also confirmed in several studies $[9,10]$. Occlusion and bilateral lesions of the carotid artery are associated with poorer neurological outcomes than stenosis and unilateral lesion in CABG [2]. These findings suggest the importance of cerebrovascular assessment from the neck to brain, including the degree of stenosis and number of lesions, in order to better gauge the risk of post-CABG stroke. Improvement in perioperative management for patients with significant cerebrovascular disease will be indispensable as a next step.

We evaluated and graded extra- and intracranial atherosclerosis prior to $\mathrm{CABG}$, and prospectively investigated whether CABG could be performed safely for patients with a high risk of postoperative stroke. For high risk patients, we adjusted blood pressure management during $C A B G$ according to the grade. We also used intraaortic balloon pump (IABP) for patients with high grades since it improves systemic perfusion even with reduced cardiac performance [11]. Fundamentally, our approach seeks to identify the degree of severity of cerebrovascular lesions for which patients can still undergo CABG with an acceptably low risk.

\section{Patients and Methods}

\subsection{Study Population}

For the present study, 200 consecutive patients were prospectively recruited to undergo sufficient cerebrovas- cular assessments prior to CABG. Whereas the vast majority of patients (198) were neurologically asymptomatic, two patients were symptomatic before CABG. Assessments included carotid echo, brain computed tomography (CT), magnetic resonance angiography (MRA) for extracranial and intracranial arteries, and head magnetic resonance imaging (MRI) for evaluation of preexisting ischemia in the brain. These assessments were generally performed in the month before CABG. When MRA was insufficient for assessment due to artifacts or other factors, 3D-CT was added as long as the patient's renal functions allowed. Brain perfusion scintigrams were also performed when necessary. Patients' baseline characteristics are shown in Table 1 . The study was approved by the institutional ethics committee.

\subsection{Cerebrovascular Grading}

Severity of cerebrovascular atherosclerosis was assessed by experienced neurosurgeons and/or radiologists who remained blind to the clinical data. The severity was based on the level of stenosis in evaluated arteries. Each stenosis was assigned one of four levels $(0,1,2,3)$ as seen below.

$<$ Extracranial Carotid and Vertebral Arteries $>$

Assessments consist of echo and MRA of the neck. The percentage of stenosis was determined by dividing the luminal diameter of the narrowest part of the artery by that of a part distal to the lesion. Level-0: $(<50 \%)$; level-1: $(50 \% \leq$ to $<70 \%)$; level-2: $(70 \% \leq$ to $<90 \%)$; level-3: $(\geq 90 \%)$.

$<$ Intracranial Carotid, Vertebral and Basilar Arteries $>$

Assessments were based on the findings of head MRA. Level assignment was done in the same fashion as above.

Table 1. Baseline characteristics in each grade.

\begin{tabular}{|c|c|c|c|c|c|c|c|}
\hline \multicolumn{8}{|c|}{$\underline{\text { Grade }}$} \\
\hline & $0(\mathrm{n}=97)$ & $1(\mathrm{n}=38)$ & $2(\mathrm{n}=29)$ & $3(n=22)$ & $4(n=14)$ & Total $(n=200)$ & $\mathrm{p}$ value \\
\hline Age & $65.0[55.0-72.3]$ & $73.0[63.0-79.0]$ & $72.0[68.0-74.0]$ & $73.0[70.0-76.0]$ & $69.0[65.0-76.0]$ & $70.0[61.0-75.0]$ & $0.0003^{*}$ \\
\hline Age $>80 y$ & $9(9.3)$ & $10(26.3)$ & $9(31.0)$ & $3(13.6)$ & $3(21.4)$ & $28(14.0 \%)$ & 0.11 \\
\hline Male & $79(81.4)$ & $28(73.7)$ & $25(86.2)$ & $19(86.3)$ & $9(64.3)$ & $160(80.0 \%)$ & 0.35 \\
\hline Hypertension & $73(75.3)$ & $32(84.2)$ & $25(86.2)$ & $19(86.3)$ & $13(92.4)$ & $162(81.0 \%)$ & 0.34 \\
\hline Diabetes Melitus & $52(53.6)$ & $18(47.4)$ & $15(51.7)$ & $16(43.5)$ & $9(74.3)$ & $110(55.0 \%)$ & 0.36 \\
\hline Hypercholesterolemia & $54(55.7)$ & $24(63.2)$ & $16(55.2)$ & $10(45.5)$ & $8(57.1)$ & $112(56.0 \%)$ & 0.77 \\
\hline Renal failure & $13(13.4)$ & $11(28.9)$ & $6(20.7)$ & $2(9.1)$ & $2(14.3)$ & $34(17.0 \%)$ & 0.19 \\
\hline Peripheral artery disease & $19(19.6)$ & $8(21.1)$ & $7(24.1)$ & $7(31.8)$ & $6(42.9)$ & $47(23.5 \%)$ & 0.32 \\
\hline LVEF & $66.0[55.8-71.5]$ & $54.0[45.8-66.3]$ & $64.0[56.3-70.5]$ & $66.5[61.0-72.0]$ & $62.0[49.8-68.5]$ & $63.5[52.5-71.0]$ & 0.07 \\
\hline LVEF < 40\% & $13(13.4)$ & $13(34.2)$ & $1(3.4)$ & $3(13.6)$ & $3(21.4)$ & $33(16.5 \%)$ & 0.5 \\
\hline Previous stroke & $6(6.2)$ & $5(13.2)$ & $1(3.4)$ & $5(22.7)$ & $4(28.6)$ & $21(10.5 \%)$ & $0.017^{\dagger}$ \\
\hline
\end{tabular}

Values are expressed as median [interquartile range] or number $(\%) .^{*}:$ Patients in grade- 0 were significantly younger than patients in grade- 1 (P $\left.<0.01\right)$, grade- $2(\mathrm{p}<0.05)$ and grade- $3(\mathrm{p}<0.05)$. $^{\dagger}$ : History of stroke tended to be more common in grade- 4 than in grade- $0(p=0.06)$. 


\section{$<$ Cerebral Arteries $>$}

Assessments were made for anterior, middle, and posterior cerebral arteries as well as Willis circle from the findings of head MRA. Level-0: normal or mild stenosis; level-1: moderate stenosis, level-2: severe stenosis; level3: occlusion. Level-0 included stenosis less than $50 \%$ as in previous studies $[8,10]$. When a flow defect was seen in a cerebral artery in one or more views in MRA, the lesion was assigned to level- 2 or 3 . To be judged as occlusion, the flow defect had to be confirmed in all the views of MRA and the length of defect had to be $5 \mathrm{~mm}$ or longer with significant decrease in the distal flow.

$<$ Cerebrovascular Grading $>$

The severity of atherosclerotic lesions in extra- and intracranial arteries was the primary factor for grading. Grade-0: no stenotic lesions greater than level-0; grade-1: patients with level-1 stenosis; grade-2: level-2 stenosis; grade-3: level-3 stenosis. Because multiple lesions bring a higher risk of postoperative stroke in CABG [2,8], patients with multiple stenosis in the same stenotic level were considered for higher grades. However, even with multiple lesions, higher grades were not assigned if the lesions did not affect the same cerebral area. For example, a patient who had level-1 stenosis at left and right anterior cerebral arteries was not increased from grade- 1 to grade-2. "Symptomatic" was also a cause for up-grading, with the aforementioned neurosurgeons making the determinations. Thus, some patients initially assigned to grade- 3 were subsequently evaluated as grade- 4 . Patients with unilateral carotid occlusion and reduced ipsilateral brain perfusion in the perfusion scintigram were also assigned grade- 4 .

Finally, plaque score of the carotid artery [12], atherosclerotic score [8], and existence of multiple brain infarctions [13] were recorded in each patient as additional assessments.

\subsection{Surgical Strategy and Intraoperative Management}

Off-pump CABG was initially adopted for all patients because it may reduce the risk of post-CABG stroke [14]. In-situ arterial graft was preferred for the same reason and for improvements in long-term graft patency. The grade of cerebrovascular lesions did not influence the number and arrangement of the grafts. Blood pressure management during surgery varied according to grade. Systolic arterial pressure (SAP) was kept at $80 \mathrm{mmHg}$ or higher throughout the operation for grade-1 and 2 patients, and $90 \mathrm{mmHg}$ or higher for grades-3 and 4 . For grade-0 patients, $70 \mathrm{mmHg}$ of SAP was the lowest allowed during distal anastomosis. Cardiac wall motion was carefully observed via transesophageal echo for all the patients, especially when SAP approached the lowest allowed level. Intra-aortic balloon pumping (IABP) was inserted approximately 2 hours before surgery for patients of grades-2, 3 and 4, and assisted the heart at a ratio of $1: 1$ throughout the operation. Positive inotropic agents were used when necessary. Landiolol was used to prevent tachycardia and atrial fibrillation for all patients [15]. Cardiopulmonary bypass was set up with cannulations onto the ascending aorta and right atrium for grade3 and 4 patients as long as there were no reasons against it. Low blood pressure in some of these patients resulted in bypass. Thiamylal sodium was used for grade- 4 patients to protect the brain $(5 \mathrm{mg} / \mathrm{kg}$ of body weight for the initial dose, followed by continuous dose of $125 \mathrm{mg} /$ hour) during surgery [16]. The ascending aorta was carefully evaluated by preoperative CT and by intraoperative echo examination when necessary. As a result, we avoided contact with the ascending aorta and used the left or right axillary artery for vein graft anastomosis and arterial cannulation for four and three patients respectively.

\subsection{Postoperative Management}

Postoperative management was carried out in the same fashion as for patients after CABG, and not different among the grades. Anti-platelet drugs were restarted the day following surgery. Warfarin was started concurrently for patients who had one or more vein grafts, while the international normalized ratio of pro-thrombin time was maintained between 1.5 and 2.0. Beta blockade was also administered to prevent atrial fibrillation [17]. Neurological problems were carefully followed throughout the postoperative course. However, brain CT and MRI were not routinely performed as long as patients were recuperating well. We observed patients for at least 30 days after surgery.

\subsection{Statistical Analyses}

All statistical analyses were performed using SPSS 16.0 J for Windows (SPSS Japan Inc, Tokyo, Japan). Because the data might not be normally distributed, nonparametric tests were used for all analyses. Difference between 2 groups was evaluated with Mann-Whitney U test. When three or more groups were compared, Kruskal-Wallis test was applied prior to Mann-Whitney $U$ test, and if the $p$ value was less than 0.05 , Bonferroni's correction was applied to the $p$ value of Mann-Whitney U test. To investigate the differences of arterial pressures in IABP study, Friedman test was performed initially and, if the $p$ value was less than 0.05, Wilcoxon test and Bonferroni's correction was applied to identify the significant differences. Spearman's test was used to investigate the correlation between cerebrovascular grading and atherosclerotic and plaque scores. A p value $<0.05$ was considered statistically significant. 


\section{Results}

\subsection{Evaluation of Cerebrovascular Atherosclerosis}

\subsubsection{Distribution and Severity of Cerebrovascular Lesions}

As shown in Table 1, significant portions of our sample suffered from diabetes mellitus $(55 \%)$, renal failure on hemodialysis (17\%), and peripheral artery disease (23.5\%). Thus, our study cohort had relatively higher risks for cerebrovascular atherosclerosis than other previous studies, perhaps resulting in a high proportion of patients with significant stenosis ( $\geq 50 \%$ ) in extra and/or intracranial arteries. As noted in Figure 1, there were 257 significant atherosclerotic lesions in 103 patients. Stenotic lesions were more common in intracranial arteries than in extracranial arteries (147 vs. 110). However, when the most severe lesion was focused in each patient, extracranial carotid artery (63 cases) was the most frequent site, followed by intracranial cerebral arteries (50 cases).

\subsubsection{Up-Grading in Cerebrovascular Evaluation}

We increased the grade of 34 of 103 patients with signif- icant atherosclerotic lesions. Details are shown in Table 2. Experienced neurosurgeons considered 14 cases (grade-4) to have an extremely high risk of stroke in this series. Details of cerebrovascular assessments in grade- 4 patients are shown in Table 3, and representative MRI images of a patient in grade- 4 are shown in Figure 2. Reduced ipsilateral brain perfusion was observed in five patients and that was a reason of up-grading for three of them. Overall, up-grades were made in $22 \%$ of grade- 1 , $23 \%$ of grade- 2 , and $33 \%$ of grade- 3 patients respectively.

\subsubsection{Other Aspects of Cerebrovascular Atherosclerosis}

Table 4 shows plaque score, atherosclerotic score, and existence of silent multiple infarctions as other indicators of cerebrovascular atherosclerosis. Note that both atherosclerotic and plaque scores shows positive correlations with grade (atherosclerotic score: $r=0.906 p<0.0001$, plaque score: $r=0.578 p<0.0001$ ), and support the hypothesis that patients in higher grades are at higher risk of neurological complications after CABG. Silent multiple infarction was significantly less in grade-0 than other 4 grades $(\mathrm{P}<0.05)$.

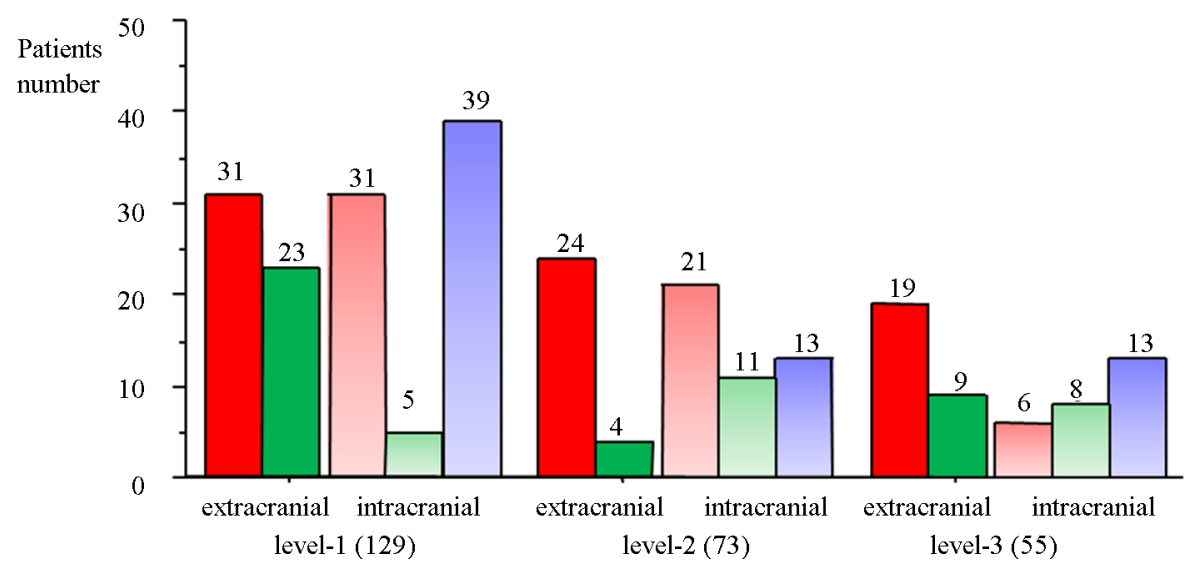

Figure 1. This figure shows distribution of atherosclerotic lesions in each stenotic level. Cerebrovascular atherosclerosis was most common at the extracranial carotid artery, followed by cerebral and intracranial carotid arteries. Total numbers of lesions were 74 in extracranial carotid artery, 58 in intracranial carotid artery, and 65 in cerebral arteries. Red bar: extracranial carotid artery; green bar: extracranial vertebral artery; red graduation bar: intracranial carotid artery; green graduation bar: intracranial vertebral and basilar arteries; blue graduation bar: cerebral artery.

Table 2. Reasons for up-grading.

\begin{tabular}{|c|c|c|c|c|c|c|c|}
\hline \multirow{2}{*}{ Grade (n) } & \multirow{2}{*}{ Up-graded patients } & \multicolumn{2}{|c|}{ Unilateral multiple lesions ${ }^{*}$} & \multicolumn{2}{|c|}{ Bilateral lesions } & \multirow{2}{*}{$\begin{array}{c}\text { Bilateral } \\
\text { multiple lesions }\end{array}$} & \multirow{2}{*}{$\begin{array}{l}\text { reduced ipsilatera } \\
\text { perfusion }^{\ddagger}\end{array}$} \\
\hline & & $\begin{array}{c}\text { Carotid - } \\
\text { ACA/MCA }\end{array}$ & $\begin{array}{l}\text { Vertebra - } \\
\text { PCA }\end{array}$ & Carotid & Vertebra & & \\
\hline $1(38)$ & 0 & 0 & 0 & 0 & 0 & 0 & 0 \\
\hline $2(29)$ & 11 & 5 & 1 & 1 & 0 & 4 & 0 \\
\hline $3(22)$ & 9 & 4 & 0 & 2 & 0 & 3 & 0 \\
\hline $4(14)$ & 14 & 4 & 1 & 0 & 0 & 6 & 3 \\
\hline
\end{tabular}

*: ACA: anterior cerebral artery, MCA: middle cerebral artery, PCA: posterior cerebral artery. ${ }^{\dagger}$ : Both carotid and vertebral lesions were included. ${ }^{\ddagger}$ : Brain perfusion was evaluated by perfusion scintigram. 
Table 3. Cerebrovascular details of grade-4 patients $(n=14)$.

1) Unilateral occlusion of extracranial carotid artery $(n=5)$

with reduced ipsilateral perfusion at rest in perfusion scintogram $(n=3)$

with reduced ipsilateral perfusion with Diamox in perfusion scintogram $(n=1)$

with symptom $(\mathrm{n}=1)$

2) Bilateral severe $(90 \%$ - $99 \%)$ stenosis of extracranial carotid artery $(n=3)$

with intracranial stenosis $(90 \%-99 \%)$ in carotid or cerebral arteries $(n=2)$

with right vertebral occlusion and multiple $70 \%-90 \%$ stenosis in left vertebral-basilar arteries $(n=1)$

3) Unilateral severe $(90 \%-99 \%)$ stenosis of extracranial carotid artery $(n=3)$

with multiple ipsilateral stenosis (level 3$)$ in intracranial carotid and/or cerebral arteries $(\mathrm{n}=2)$

with multiple intracranial carotid stenosis (level 3$)$ and reduced ipsilateral perfusion $(n=1)$

4) Severe vertebral and basilar arterial lesions $(n=3)$

bilateral vertebral occlusions with poor collateral to basilar artery $(\mathrm{n}=1)$

bilateral vertebral occlusions, and multiple severe (level 3) stenosis in cerebral arteries ( $\mathrm{n}=1)$

left vertebral occlusion and $90 \%$ stenosis of right vertebral artery with poor collateral to basilar artery $(\mathrm{n}=1)$

(A)

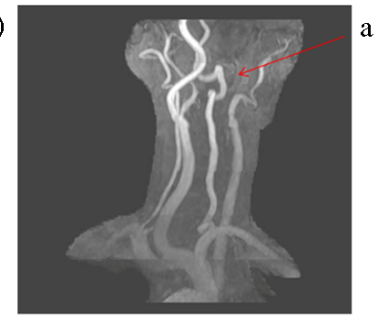

(B)

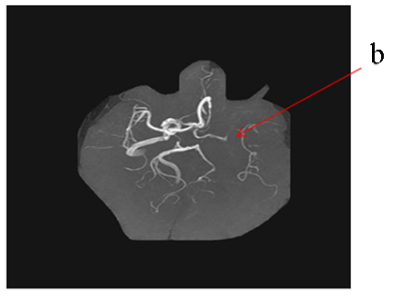

(C)

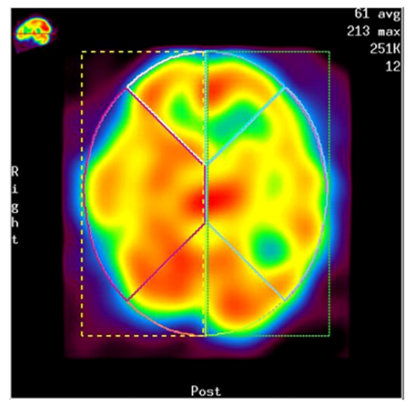

Figure 2. Cerebrovascular findings of a patient in grade-4. (A) The left internal carotid artery was occluded (allow-a) in cervical magnetic resonance angiography (MRA). (B) The left anterior and middle cerebral arteries were not apperared (allow-b) in intracranial MRA. Communication via Willis circle was poor. (C) Brain perfusion scintigram of this patient showed reduced blood flow on the left side.

Table 4. Other cerebrovascular findings.

\begin{tabular}{cccccccc}
\hline \multicolumn{7}{c}{$\underline{\text { Grade }}$} \\
\hline & $0(\mathrm{n}=97)$ & $1(\mathrm{n}=38)$ & $2(\mathrm{n}=29)$ & $3(\mathrm{n}=22)$ & $4(\mathrm{n}=14)$ & Total $(\mathrm{n}=200)$ & $\mathrm{p}$ value \\
\hline Carotid plaque score & $6.0[3.0-9.5]$ & $9.7[6.0-12.9]$ & $11.4[8.0-17.0]$ & $14.3[11.0-18.9] 14.6[11.4-20.0]$ & $9.0[5.0-12.9]$ & $<0.0001^{*}$ \\
Atherosclerotic score & $0.0[0.0-0.0]$ & $1.0[1.0-1.0]$ & $2.0[1.8-3.0]$ & $3.0[2.0-4.0]$ & $5.0[4.0-6.0]$ & $1.0[0.0-2.0]$ & $<0.0001^{\dagger}$ \\
Silent multiple infarction & $17(17.5)$ & $19(50.0)$ & $17(58.6)$ & $11(50.0)$ & $8(57.1)$ & $72(36.0)$ & $0.0002^{\ddagger}$ \\
\hline
\end{tabular}

Values are expressed as median [interquartile range] or number (\%). ": Patients in grade 0 showed significantly lower plaque scores than the other four grades $(\mathrm{p}$ $<0.001)$. Plaque scores were significantly lower in grade 1 than in grades 3 and $4(\mathrm{P}<0.05)$. ${ }^{\dagger}$ : When any two of the five grades were compared, atherosclerotic score was always significantly higher in the higher grade $(\mathrm{p}<0.001)$ except between grades 2 and $4(\mathrm{p}=0.13)$. : Silent multiple infarction was significantly less common in grade 0 than the other grades $(\mathrm{P}<0.05)$. 


\subsection{Clinical Outcomes}

\subsubsection{Imtraoperative Parameters and Postoperative Course}

Intraoperative parameters and clinical outcomes in each grade are shown in Table 5. Complete revascularization was achieved in all patients with distal anastomosis of 4.0 on average. It was natural that the use of IABP was significantly more frequent in grades-2, 3 and 4 than in grades- 0 and 1 . Unscheduled insertion of IABP during surgery due to low blood pressure was done for two grade-1 patients. There were no significant differences in usage of cardiopulmonary bypass among the grades. Two grade- 4 cases necessitated cardiopulmonary bypass because their systolic blood pressures could not be kept at $90 \mathrm{mmHg}$ during anastomosis. Once cardiopulmonary bypass started, we easily maintained systolic blood pressure higher than $100 \mathrm{mmHg}$ with sufficient pulsatile patterns for both patients, allowing us to perform coronary anastomosis under stable hemodynamic conditions. There were no significant differences among the grades in ventilation time, intensive care unit (ICU) stay, and hospital stay.

\subsubsection{Neurological Complications}

Neurological complications are shown in Table 5. All the patients recovered consciousness without neurological complications in ICU. Postoperative stroke was observed in one patient, a 75-year-old man who was in grade-2 due to stenosis of the extra-cranial right carotid artery and had an uneventful course until the stroke occurred on the fifth day after CABG. His only symptom was a mild speaking disturbance and the focus was at the left internal capsule. A small $(<1 \mathrm{~cm})$ focal ischemic change was revealed by MRI. The patient gradually improved under medical treatment and his speech was near normal by the time he was discharged from the hospital. Two other patients also displayed transient symptoms. One patient had a convulsion that lasted for only $5-6$ seconds and the other showed an involuntary movement in his hands. Both were happened on the third day from CABG with no new ischemic changes in their brain CT and MRI. No additional treatment was required for these events.

\section{Discussion}

In the present study, we evaluated cerebrovascular lesions fully from the neck to brain. For the first time, we developed a grading system for the lesions to determine efficacious management in surgery. Our grading is not just a degree of steno-occlusive change in certain arteries

Table 5. Surgical details and clinical outcomes.

\begin{tabular}{|c|c|c|c|c|c|c|c|c|}
\hline \multicolumn{9}{|c|}{$\underline{\text { Grade }}$} \\
\hline & & $0(\mathrm{n}=97)$ & $1(\mathrm{n}=38)$ & $2(n=29)$ & $3(n=22)$ & $4(n=14)$ & Total & $\mathrm{p}$ value \\
\hline \multicolumn{2}{|c|}{ No of distal anastomosis } & $4.0[2.0-5.0]$ & $3.5[2.0-4.0]$ & $5.0[3.0-5.0]$ & $4.0[3.0-6.0]$ & $4.0[3.0-4.0]$ & $4.0[3.0-5.0]$ & 0.13 \\
\hline \multicolumn{2}{|c|}{ Use of IABP* } & $13(13.4)$ & $6(15.8)$ & $21(72.4)$ & $18(81.3)$ & $12(86.7)$ & $70(35.0)$ & $<0.0001^{\ddagger}$ \\
\hline \multicolumn{2}{|c|}{ Use of $\mathrm{CPB}^{\dagger}$} & $3(3.1)$ & $1(2.6)$ & $0(0.0)$ & $0(0.0)$ & $2(14.3)$ & $6(3.0)$ & 0.10 \\
\hline \multicolumn{2}{|c|}{ Ventilation time (hrs) } & $8.0[4.0-16.0]$ & $10.5[6.0-20.0]$ & $8.0[5.0-16.0]$ & $11.5[5.0-15.0]$ & $9.5[6.0-16.0]$ & $9.0[4.0-16.0]$ & 0.60 \\
\hline \multicolumn{2}{|c|}{ ICU stay (days) } & $3.0[2.0-4.0]$ & $3.5[2.0-6.0]$ & $3.0[2.0-4.0]$ & $3.0[2.0-5.0]$ & $4.0[4.0-5.0]$ & $3.0[2.0-4.0]$ & $0.026^{\S}$ \\
\hline \multicolumn{2}{|c|}{ Hospital stay (days) } & \multicolumn{6}{|c|}{$16.0[13.0$ - 23.0]21.5[17.5 - 32.5] $18.0[14.0-26.0] 26.5[18.0-42.0] 32.0[17.0-36.5] 19.0[14.0-30.0]$} & $0.008^{\prime}$ \\
\hline \multicolumn{9}{|c|}{ Postoperative neurological events } \\
\hline \multirow[t]{3}{*}{ Transient } & Immediate & 0 & 0 & 0 & 0 & 0 & $0(0.0)$ & \\
\hline & Delayed & 0 & 1 & 1 & 0 & 0 & $2(1.0)$ & \\
\hline & Total & $0(0.0)$ & $1(2.6)$ & $1(3.4)$ & $0(0.0)$ & $0(0.0)$ & $2(1.0)$ & 0.39 \\
\hline \multirow[t]{3}{*}{ Stroke } & Immediate & 0 & 0 & 0 & 0 & 0 & $0(0.0)$ & \\
\hline & Delayed & 0 & 0 & 1 & 0 & 0 & $1(0.5)$ & \\
\hline & Total & $0(0.0)$ & $0(0.0)$ & $1(3.4)$ & $0(0.0)$ & $0(0.0)$ & $1(0.5)$ & 0.21 \\
\hline \multicolumn{2}{|c|}{ Total } & $0(0.0)$ & $1(2.6)$ & $2(6.8)$ & $0(0.0)$ & $0(0.0)$ & $3(1.5)$ & 0.09 \\
\hline \multicolumn{2}{|c|}{ Hospital death } & $0(0.0)$ & $1(2.6)$ & $0(0.0)$ & $0(0.0)$ & $0(0.0)$ & $1(0.5)$ & 0.37 \\
\hline
\end{tabular}

Values are expressed as median [interquartile range] or number (\%). ${ }^{*}:$ Intra aortic balloon pump, ${ }^{\dagger}:$ Cardiopulmonary bypass, ${ }^{\dagger}:$ Use of IABP was significantly more common in grades 2,3 and 4 than in grade 0 and $1(\mathrm{p}<0.001) .{ }^{\S}$ : There were no significant differences between any two grades among the five grades. ": Patients in grade 3 tended to have longer hospital stay than patients in grade $0(p=0.06)$. 
or an amount of those lesions, but evaluates a risk of regional ischemia in the whole brain. Many patients with high risks for atherosclerosis were in our cohort, and experienced neurosurgeons considered 14 cases (7\%) to have an extremely high risk of stroke. We planned CABG for all these patients without pursuing surgical treatment of brain perfusion ahead of time, and successfully carried out the protocols with satisfactory outcomes.

Off-pump CABG has the potential benefit of preventing perioperative stroke [14]. However, it is known that heart displacement for distal anastomosis can cause significant drops in cardiac performance and cerebral perfusion [18]. Because decreases in cerebral oxygen saturation and hemodynamic parameters during heart displacement were correlated in previous studies [19], we determined the targeted lowest SAP for each grade (raising it accordance with up-grading) in order to maintain cerebral blood flow. It is known that cerebral blood flow is determined by autoregulatory mechanisms between the mean arterial pressures of $50 \mathrm{mmHg}$ and $150 \mathrm{mmHg}$ [20]. In the present study, when the systolic arterial pressures were about 80 and $90 \mathrm{mmHg}$, the mean arterial pressures were $57 \mathrm{mmHg}$ and $64 \mathrm{mmHg}$ on average respectively. Prior to the commencement of this study, we collaborated with neurosurgeons to determine the targeted lowest SAP for the maintenance of cerebral blood flow.

Beneficial effects of IABP on hemodynamic parameters and clinical outcome in off-pump CABG with high risks have been reported in several recent studies [11]. In these reports, prophylactic IABP increased cardiac index after two hours from insertion and significantly reduced perioperative inotropic support. We predicted the advantage of IABP for brain perfusion in conditions of heart displacement during off-pump surgery and adopted it for patients with severe stenosis in preoperative assessment (grades-2, 3, and 4). Although it was difficult to evaluate the numerical value of IABP's effect in each patient, peak arterial pressure increased approximately $10 \mathrm{mmHg}$ with IABP when systolic arterial pressure was around 80 $\mathrm{mmHg}$, which is potentially equivalent to $90 \mathrm{mmHg}$ of systolic arterial pressure without IABP. Therefore, if conditions were not conducive for IABP use for patients in grade-2 or higher, systolic arterial pressure was maintained at $90 \mathrm{mmHg}$ or higher for grade- 2 and $100 \mathrm{mmHg}$ or higher for grades-3 and 4.

Six patients $(3 \%)$ were put on cardiopulmonary bypass. Two of them were grade 4 with insufficient systemic perfusion as the reason for cardiopulmonary bypass. Because cardiopulmonary bypass was already set up for five of six cases (one was acute conversion), starting the bypass went swiftly and smoothly even while performing anastomosis. Although some surgeons and scientists may argue against cardiopulmonary bypass, we believe by- pass is an adequate procedure that can bring great benefit to the patient in this setting in terms of stroke prevention. Systemic perfusion dramatically improved for all of such patients in this study. Our findings indicate that cardiopulmonary bypass achieves the vital goal of maintaining sufficient and pulsatile flow in the brain.

We observed three neurological events, including one stroke in this series. The stroke occurred in a grade- 2 case on the opposite side of his carotid arterial stenosis. The stroke was not related to low blood pressure (less than $100 \mathrm{mmHg}$ of systolic pressure) or atrial fibrillation. Since the patient had a silent multiple infarction, a risk factor for stroke according to previous studies [13], and the area of his stroke was very small like lacunar infarction, the same mechanism might be at work in this case. Because $40 \%-60 \%$ of post-CABG strokes have been reported as a delayed stroke like this case [7], further efforts are necessary to reduce this type of stroke.

\section{Limitations of the Study}

Firstly, we did not evaluate subclinical neurological complications after CABG. Additionally, progression of dementia was not routinely evaluated by experts, although patients' families and we did not notice the significant progression in any patient. Regarding sample size, the number of patients in each grade was relatively small, especially among the higher grades, suggesting the need for further investigations. Finally, all patients were ethnically Japanese and treated by single surgical team, introducing the possibilities of ethnic-and surgeon-based biases.

\section{Conclusion}

We extensively evaluated cerebral atherosclerosis with expert support for consecutive 200 patients and successfully performed isolated CABG with a satisfactorily low incidence of neurological complications, even for patients with severe cerebrovascular disease. We argue that CABG can be safely performed without preceding or combined surgical treatment of cerebrovascular disease, but rather with sufficient preoperative cerebrovascular assessment, management of blood pressure during surgery, and postoperative medical treatment.

\section{REFERENCES}

[1] G. W. Roach, M. Kanchuger, C. M. Mangano, M. Newman, N. Nussmeier, R. Wolman, et al., "Adverse Cerebral Outcomes after Coronary Artery Bypass Surgery. Multicenter Study of Perioperative Ischemia Research Group and the Ischemia Research and Education Foundation Investigators," The New England Journal of Medicine, Vol. 335, No. 25, 1996, pp. 1857-1863.

http://dx.doi.org/10.1056/NEJM199612193352501 
[2] A. R. Naylor, Z. Mehta, P. M. Rothwell and P. R. F. Bell, "Carotid Artery Disease and Stroke during Coronary Artery Bypass: A Critical Review of the Literature," European Journal of Vascular and Endovascular Surgery, Vol. 23, No. 4, 2002, pp. 283-294. http://dx.doi.org/10.1053/ejvs.2002.1609

[3] A. R. Naylor, R. L. Cuffe, P. M. Rothwell and P. R. F. Bell, "A Systematic Review of Outcomes Following Staged and Synchronous Carotid Endarterectomy and Coronary Artery Bypass," European Journal of Vascular and Endovascular Surgery, Vol. 25, No. 5, 2003, pp. 380-389. http://dx.doi.org/10.1053/ejvs.2002.1895

[4] S. Manabe, T. Shimokawa, T. Fukui, K. Fumimoto, N. Ozawa, H. Seki, et al., "Influence of Carotid Artery Stenosis on Stroke in Patients Undergoing Off-Pump Coronary Artery Bypass Grafting," European Journal of Vascular and Endovascular Surgery, Vol. 34, No. 5, 2008, pp. 1005-1008. http://dx.doi.org/10.1016/j.ejcts.2008.08.005

[5] S. Venkatachalam, B. H. Gray, D. Mukherjee and M. H. Shishehbor, "Contemporary Management of Concomitant Carotid and Coronary Artery Disease," Heart, Vol. 97, No. 3, 2011, pp. 175-180. http://dx.doi.org/10.1136/hrt.2010.203612

[6] A. R. Naylor, "Does the Risk of Post-CABG Stroke Merit Staged or Synchronous Reconstruction in Patients with Symptomatic or Asymptomatic Carotid Disease?" The Journal of Cardiovascular Surgery, Vol. 50, No. 1, 2009 , pp. 71-81.

[7] P. Kolh, W. Wijns, N. Danchin, C. D. Mario, V. Falk, T. Folliguet, et al., "Guidelines on Myocardial Revascularization. The Task Force on Myocardial Revascularization of the European Society of Cardiology (ESAC) and the European Association of the Cardio-Thoracic Surgery (EACTS)," European Journal Cardio-Thoracic Surgery, Vol. 38, 2010, pp. S1-S52.

http://dx.doi.org/10.1016/j.ejcts.2010.08.019

[8] E. J. Lee, K. H. Choi, J. S. Ryu, S. B. Jeon, S. W. Lee, S. W. Park, et al., "Stroke Risk after Coronary Artery Bypass Graft Surgery and Extent of Cerebral Artery Atherosclerosis," Journal of the American College of Cardiology, Vol. 57, No. 18, 2011, pp. 1811-1818.

http://dx.doi.org/10.1016/j.jacc.2010.12.026

[9] S. Ohuchi, K. Kawazoe, H. Izumoto and K. Yoshioka, "Head and Neck Magnetic Resonance Angiography before Coronary Artery Bypass Grafting," Surgery Today, Vol. 35, No. 6, 2005, pp. 432-435.

http://dx.doi.org/10.1007/s00595-004-2973-4

[10] K. Uekita, N. Hasebe, N. Funayama, H. Aoyama, K. Kuroda, H. Aizawa, et al., "Cervical and Intracranial Atherosclerosis and Silent Brain Infarction in Japanese Patients with Coronary Artery Disease," Cerebrovascular Diseases, Vol. 16, No. 1, 2003, pp. 61-68. http://dx.doi.org/10.1159/000070117

[11] P. Y. Etienne, S. Papadatos, D. Glineur, Y. Mairy, E. E. Khoury, P. Noirhomme, et al., "Reduced Mortality in High-
Risk Coronary Patients Operated off Pump with Preoperative Intraaortic Balloon Counterpulsation," The Annals of Thoracic Surgery, Vol. 84, No. 2, 2007, pp. 498-503. http://dx.doi.org/10.1016/j.athoracsur.2007.03.080

[12] T. Wannarong, G. Parraga, D. Buchanan, A. Fenster, A. A. House, D. G. Hackam, et al., "Progression of Carotid Plaque Volume Predicts Cardiovascular Events," Stroke, Vol. 44, No. 7, 2013, pp. 1859-1865. http://dx.doi.org/10.1161/STROKEAHA.113.001461

[13] A. Ito, T. Goto, K. Maekawa, T. Baba, Y. Mishima and K. Ushijima, "Postoperative Neurological Complications and Risk Factors for Pre-Existing Silent Brain Infarction in Elderly Patients Undergoing Coronary Artery Bypass Grafting," Journal of Anesthesia, Vol. 26, No. 3, 2012, pp. 405-411. http://dx.doi.org/10.1007/s00540-012-1327-4

[14] R. Pawlaczyk, D. Swietlik, R. Lango and J. Rogowski, "Off-Pump Coronary Surgery May Reduce Stroke, Respiratory Failure, and Mortality in Octogenarians," The Annals of Thoracic Surgery, Vol. 94, No. 1, 2012, pp. 29-37. http://dx.doi.org/10.1016/j.athoracsur.2012.03.037

[15] K. Nakanishi, S. Takeda, C. Kim, S. Kohda and A. Sakamoto, "Postoperative Atrial Fibrillation in Patients Undergoing Coronary Artery Bypass Grafting or Cardiac Valve Surgery: Intraoperative Use of Landiolol," Journal of Cardiothoracic Surgery, Vol. 8, No. 1, 2013, pp. 19-25. http://dx.doi.org/10.1186/1749-8090-8-19

[16] R. Z. Zhan, N. Fujiwara, T. Yamakura, K. Taga, S. Fukuda and K. Shimoji, "Differential Inhibitory Effects of Thiopental, Thiamylal and Phenobarbital on Both Voltage-Gated Calcium Channels and NMDA Receptors in Rat Hoppocampal Slices," British Journal of Anaesthesia, Vol. 81, No. 6, 1998, pp. 932-939. http://dx.doi.org/10.1093/bja/81.6.932

[17] I. Yoshioka, M. Sakurai, A. Namai and T. Kawamura, "Postoperative Treatment of Carvedilol Following Low Dose Landiolol Has Preventive Effect for Atrial Fibrillation after Coronary Artery Bypass Grafting," The Thoracic and Cardiovascular Surgeon, Vol. 57, No. 8, 2009, pp. 464-467. http://dx.doi.org/10.1055/s-0029-1186069

[18] S. Moritz, J. Rochon, S. Völkel, M. Hilker, J. Hobbhahn, B. M. Graf, et al., "Determinants of Cerebral Oximetry in Patients Undergoing Off-Pump Coronary Artery Bypass Grafting: An Observational Study," European Journal of Anaesthesiology, Vol. 27, No. 6, 2010, pp. 542-549.

[19] B. Atasever, C. Boer, R. Speekenbrink, J. Seyffert, P. Goedhart, B. D. Mol, et al., "Cardiac Displacement during Off-Pump Coronary Artery Bypass Grafting Surgery: effect on Sublingual Microcirculation and Cerebral Oxygenation," Interactive Cardiovascular and Thoracic Surgery, Vol. 13, No. 6, 2011, pp. 573-578. http://dx.doi.org/10.1510/icvts.2011.272047

[20] P. K. Smith, "Predicting and Preventing Adverse Neurologic Outcomes with Cardiac Surgery," Journal of Cardiac Surgery, Vol. 21, No. Suppl 1, 2006, pp. S15-S19. http://dx.doi.org/10.1111/j.1540-8191.2006.00215.x 